\title{
Secret Image Sharing for General Access Structures using Random Grids
}

\author{
Sachin Kumar \\ Department of Mathematics \\ Indian Institute of Technology Delhi \\ Hauz Khas, New Delhi - 110016, India
}

\author{
R. K. Sharma \\ Department of Mathematics \\ Indian Institute of Technology Delhi \\ Hauz Khas, New Delhi - 110016, India
}

\begin{abstract}
This paper presents a visual secret sharing (VSS) scheme for general access structures by using random grids. Compared to the existing VSS schemes for general access structures, the proposed scheme generates the shares of same size as that of the original secret image and does not require any codebook prior to encryption process. With these advantages, the proposed scheme broadens the potential applicability of random-grid based VSS. We prove that the proposed scheme gives the strong access structure. Formal proofs, security analysis and experimental results are given to demonstrate both the feasibility and the correctness of the proposed scheme.
\end{abstract}

\section{General Terms:}

Cryptography, Information Security

\section{Keywords:}

Visual secret sharing, Visual cryptography, Random grids, General access structure, Image encryption

\section{INTRODUCTION}

Shamir [12] and Blakley [3] independently proposed $(k, n)$ threshold secret sharing (SS) scheme in which a secret is shared among $n$ participants such that the secret can be obtained by at least $k(\leq n)$ participants together, but any $k-1$ or fewer participants cannot obtain any secret information. The SS schemes [3, 12] reconstruct the secret accurately by using complex computation. To share a visual secret information, several visual secret sharing (VSS) schemes [4, 9, 11, 13, 14, 16] are developed involving complex, little or no computation in the decryption phase.

In 1995, Naor and Shamir [11] proposed a new technique known as Visual Cryptography (VC), which shares a visual information and removes the problem of computation involved in the decryption phase. Naor and Shamir's scheme is a $(k, n)$-threshold scheme, which encrypts a black and white secret image into $n$ meaningless shares such that knowledge of less than $k$ shares reveals nothing about the secret image. However, the secret image can be reconstructed by xeroxing at least $k(\leq n)$ shares on transparencies and stacking these transparencies together. The reconstruction is performed by human visual system without any computation. A VC scheme for an access structure splits the secret image into a set of shares such that certain qualified set of participants can visually recover the secret image, but other forbidden set of participants have no information about the secret image. The different construction techniques of VC scheme for general access structures, where an access structure is a specification of all qualified and forbidden sets of participants, were studied in [1, 2]. These schemes generate the shares of the size larger than that of the secret and require collections of basis matrices (codebook) prior to encryption process. In 1987, Kafri and Keren [6] proposed a random-grid based $(2,2)$ VSS technique in which a binary secret image is encrypted into two cipher grids without any pixel expansion and codebook requirement. The decryption is same as in traditional VC. Shyu [13] extended Kafri and Keren's scheme to $(n, n)$ scheme for any $n(\geq 2)$. Chen [4] also proposed $(2, n)$ and $(n, n)$ VSS schemes based on random grids. Further, Chen and Tsao [5] proposed a random-grid based $(k, n)$-threshold VSS scheme, which is limited to threshold access structure and cannot be used for general access structures. Wu and Sun [16] developed a VSS scheme for general access structures. In their scheme original basis matrices, generated by the conventional VC scheme for general access structures [2], are modified to generate the shares. However, Wu and Sun's scheme has no pixel expansion but requires to generate basis matrices prior to encryption process. Recently, Shyu [14] proposed another VSS technique for general access structures without any pixel expansion and codebook requirement. They gave two construction techniques, where one uses the basis and other uses the collection of maximal forbidden sets to generate a set of visual cryptograms of random grids. For a given access structure, both the construction techniques can yield the reconstructed images of different quality.

In this paper, a random-grid based VSS scheme for general access structures is designed having the following merits.

1. No pixel expansion - The size of each share is same as that of the original image. It makes the storage and distribution of shares more efficient.

2. No codebook requirement - The proposed scheme does not require the collections of basis matrices for generating shares.

3. Generalized - The proposed scheme generalizes the existing random-grid based VSS schemes to general access structures.

4. Wide image format - The proposed scheme can be used to encrypt binary as well as color images.

The rest of this paper is organized as follows. Section 2 reviews VC schemes for general access structures, and the traditional randomgrid based VSS. Section 3 presents the proposed scheme for binary 
and color images. The security and performance analysis of the proposed scheme are discussed in Section 4. Section 5 presents the experimental results and comparison with related work. Finally, the paper is concluded in Section 6.

\section{PRELIMINARIES}

This section presents the results from VC schemes for general access structures and discusses traditional random-grid based VSS.

\subsection{Review of VC schemes for general access structures}

Let $\mathcal{P}=\{1,2, \ldots, n\}$ be a set of $n$ participants, and $2^{\mathcal{P}}$ denote the set of all subsets of $\mathcal{P}$. Let $\Gamma_{\text {Qual }} \subseteq 2^{\mathcal{P}}$ and $\Gamma_{\text {Forb }} \subseteq 2^{\mathcal{P}}$, where $\Gamma_{\text {Qual }} \cap \Gamma_{\text {Forb }}=\emptyset$. The members of $\Gamma_{Q u a l}$ are referred as qualified sets, while the members of $\Gamma_{F o r b}$ are referred as forbidden sets. The pair $\left(\Gamma_{Q u a l}, \Gamma_{F o r b}\right)$ is known as the access structure.

We define $\Gamma_{0}$ as a set consisting of the minimal qualified sets, i.e., $\Gamma_{0}=\left\{Q \in \Gamma_{\text {Qual }}: Q^{\prime} \notin \Gamma_{Q u a l}, \forall Q^{\prime} \subset Q\right\}$. A monotone increasing (respectively monotone decreasing) access structure $\Gamma$ on $\mathcal{P}$ is a subset $\Gamma \subseteq 2^{\mathcal{P}} \backslash \emptyset\left(\Gamma \subseteq 2^{\mathcal{P}}\right)$ such that if $Q \in \Gamma$ and $Q \subseteq Q^{\prime} \subseteq \mathcal{P}$ (respectively $Q^{\prime} \subseteq Q \subseteq \mathcal{P}$ ), then $Q^{\prime} \in \Gamma$. If $\Gamma_{Q u a l}$ is monotone increasing, $\Gamma_{F o r b}$ is monotone decreasing, and $\Gamma_{Q u a l} \cup \Gamma_{F o r b}=2^{\mathcal{P}}$ then the access structure is called strong access structure and $\Gamma_{0}$ is called the basis of the access structure,.

Ateniese et al. [2] were the first to consider VC-based VSS for general access structures. They proposed two constructions for binary images. The first construction uses the cumulative array method to generate shares, while in the second construction smaller schemes are used as building blocks in construction of larger schemes. In [2], both constructions give strong access structure and obtain the pixel expansion as presented by the following results.

Result 1: Let $\left(\Gamma_{Q u a l}, \Gamma_{F o r b}\right)$ be a strong access structure. Any set $F \in \Gamma_{\text {Forb }}$ is said to be maximal forbidden if $F \cup\{p\} \in \Gamma_{\text {Qual }}$ for all $p \in \mathcal{P} \backslash F$. Let $Z_{M}$ denote the collection of the maximal forbidden sets in $\Gamma_{F o r b}$. Then, for the given access structure, there exists a VC scheme having pixel expansion equals to $2^{\left|Z_{M}\right|-1}$.

Result 2: Let $\left(\Gamma_{Q u a l}, \Gamma_{\text {Forb }}\right)$ be a strong access structure with the basis $\Gamma_{0}$. Then, for the given access structure, there exists a VC scheme having pixel expansion equals to $\sum_{X \in \Gamma_{0}} 2^{|X|-1}$. Adhikari et al. [1] proposed another black and white VC for strong access structures, where basis matrices are constructed by using the fact that the collection of all solutions of a system of linear homogeneous equations over the binary field forms a vector space over the base field.

\subsection{Review of traditional random-grid based VSS}

A random grid is defined as a transparency comprising a twodimensional array of pixels, where each pixel is either transparent (0) or opaque (1), chosen randomly similar to a coin-flip procedure. Kafri and Keren [6] proposed three algorithms to encrypt a binary image into two cipher grids, which are regarded as Algorithms 1-3.

Input: Binary secret image $A$ of the size $h \times w$.

Output: Cipher grids $R_{1}$ and $R_{2}$ of the size $h \times w$.

\section{Algorithm 1}

Step 1.1 Generate $R_{1}$ randomly, i.e., $R_{1}[i, j]=\operatorname{random}(0,1)$, where $1 \leq i \leq h$ and $1 \leq j \leq w$
Step 1.2 Generate $R_{2}$ by $R_{1}$ and $A$ as follows

for (each pixel $A[i, j], 1 \leq i \leq h$ and $1 \leq j \leq w$ )

\{

\}

$$
\begin{aligned}
& \text { if }(A[i, j]=0) R_{2}[i, j]=R_{1}[i, j] \\
& \text { else } R_{2}[i, j]=\overline{R_{1}[i, j]}
\end{aligned}
$$

Step 1.3 output $\left(R_{1}, R_{2}\right)$

\section{Algorithm 2}

Step 2.1 Generate $R_{1}$ randomly, i.e., $R_{1}[i, j]=\operatorname{random}(0,1)$, where $1 \leq i \leq h$ and $1 \leq j \leq w$

Step 2.2 Generate $R_{2}$ by $R_{1}$ and $A$ as follows for (each pixel $A[i, j], 1 \leq i \leq h$ and $1 \leq j \leq w$ ) \{

$$
\begin{aligned}
& \text { if }(A[i, j]=0) R_{2}[i, j]=R_{1}[i, j] \\
& \text { else } R_{2}[i, j]=\operatorname{random}(0,1)
\end{aligned}
$$$$
\}
$$

Step 2.3 output $\left(R_{1}, R_{2}\right)$

\section{Algorithm 3}

Step 3.1 Generate $R_{1}$ randomly, i.e., $R_{1}[i, j]=\operatorname{random}(0,1)$, where $1 \leq i \leq h$ and $1 \leq j \leq w$

Step 3.2 Generate $R_{2}$ by $R_{1}$ and $A$ as follows for (each pixel $A[i, j], 1 \leq i \leq h$ and $1 \leq j \leq w$ ) \{

$$
\begin{aligned}
& \text { if }(A[i, j]=0) R_{2}[i, j]=\operatorname{random}(0,1) \\
& \text { else } R_{2}[i, j]=\overline{R_{1}[i, j]}
\end{aligned}
$$

Step 3.3 output $\left(R_{1}, R_{2}\right)$

$\operatorname{random}(0,1)$ is a function that returns a value $(0$ or 1$)$ randomly $\bar{R}$ is defined as an inverse grid of a binary grid $R$ of the size $h \times w$ obtained by bitwise complementing of $R$, i.e., $\overline{R[i, j]}=1-R[i, j]$ for $1 \leq i \leq h$ and $1 \leq j \leq w$. The cipher grids $R_{1}$ and $R_{2}$ do not reveal any secret information. However, the image obtained by stacking $R_{1}$ and $R_{2}$ visually reveals the secret image.

\section{THE PROPOSED SCHEME}

This section presents the proposed scheme for general access structures based on random grids. The proposed scheme encrypts a secret image into $n(\geq 2)$ cipher grids such that each cipher grid is meaningless, and stacking result of the cipher grids corresponding to participants of any forbidden set reveals no secret information. However, the secret image can be recognized visually by stacking the cipher grids corresponding to participants of any qualified set.

\subsection{Scheme for binary images}

Some notations are given before presenting the proposed scheme. Let $\left(\Gamma_{\text {Qual }}, \Gamma_{\text {Forb }}\right)$ be a strong access structure defined on a set of $n$ participants $\mathcal{P}=\{1,2, \ldots, n\}$ with the basis $\Gamma_{0}$. Let $\left|\Gamma_{0}\right|=N$ and $\Gamma_{0}$ contains the subsets of $\mathcal{P}$ of different cardinalities $m_{1}, m_{2}, \ldots, m_{r}$, where $m_{1}<m_{2}<\ldots<m_{r}$ and $m_{j} \in\{2,3, \ldots, n\}$ for $1 \leq j \leq r$. Let $n_{j}(1 \leq j \leq r)$ denote the number of subsets of cardinality $m_{j}$ in $\Gamma_{0}$, where $n_{j} \in\left\{1,2, \ldots,\left(\begin{array}{c}n \\ m_{j}\end{array}\right)\right\}$ and $n_{1}+n_{2}+\ldots+n_{r}=N$. We define $\Gamma_{0}=\left\{B_{m_{1}}^{1}, \ldots, B_{m_{1}}^{n_{1}}, B_{m_{2}}^{1}, \ldots, B_{m_{2}}^{n_{2}}, \ldots, B_{m_{r}}^{1}, \ldots, B_{m_{r}}^{n_{r}}\right\}$, where $B_{m_{k}}^{t}$ denotes $t^{t h}$ subset of cardinality $m_{k}, 1 \leq t \leq n_{k}$ and $1 \leq k \leq r$. Let $B_{m_{k}}^{t}$ be the set of participants $p_{1}^{t}, p_{2}^{t}, \ldots, p_{m_{k}}^{t} \in\{1,2, \ldots, n\}$, i.e., $B_{m_{k}}^{t}=\left\{p_{1}^{t}, p_{2}^{t}, \ldots, p_{m_{k}}^{t}\right\}$. The 
procedure for sharing a secret image into $n$ cipher grids for any general access structure is given in Algorithm 4. Here, $\oplus$ denotes the Boolean exclusive OR operation.

Input: Binary secret image $A$ of the size $h \times w$ and a strong access structure $\left(\Gamma_{\text {Qual }}, \Gamma_{\text {Forb }}\right)$ with the basis $\Gamma_{0}$.

Output: Cipher grids $R_{1}, R_{2}, \ldots, R_{n}$ of the size $h \times w$.

\section{Algorithm 4}

Step 4.1 Select a pixel $A[i, j] \in A$ and encrypt it into $n$ random values $R_{1}[i, j], R_{2}[i, j], \ldots, R_{n}[i, j]$ by following Steps 4.2 to 4.6

Step 4.2 Select randomly one set from the basis $\Gamma_{0}$. Let us assume that the selected set is $B_{m_{k}}^{t}$, where $t \in\left\{1,2, \ldots, n_{k}\right\}$ and $k \in$ $\{1,2, \ldots, r\}$

Step 4.3 For the selected pixel $A[i, j]$ generate binary values $a_{1}, a_{2}, \ldots, a_{m_{k}}$ by using the traditional random-grid based VSS as follows

Step 4.3.1 Generate $a_{1}, a_{2}, \ldots, a_{m_{k}-1}$ independently by the function random $(0,1)$

Step 4.3.2 Select one algorithm from Algorithms 1-3 and generate $a_{m_{k}}$ from $a_{1}, a_{2}, \ldots, a_{m_{k}-1}$ as follows

$b_{1}=a_{1}$

for $\left(2 \leq l \leq m_{k}-1\right)$

\{

\}

$$
b_{l}=b_{l-1} \oplus a_{l}
$$

Case 1: Algorithm 1 is selected if $(A[i, j]=0) a_{m_{k}}=b_{m_{k}-1}$ else $a_{m_{k}}=\overline{b_{m_{k}-1}}$

Case 2: Algorithm 2 is selected if $(A[i, j]=0) a_{m_{k}}=b_{m_{k}-1}$ else $a_{m_{k}}=\operatorname{random}(0,1)$

Case 3: Algorithm 3 is selected if $(A[i, j]=0) a_{m_{k}}=\operatorname{random}(0,1)$ else $a_{m_{k}}=\overline{b_{m_{k}-1}}$

Step 4.4 Generate the binary values $a_{m_{k+1}}, a_{m_{k+2}}, \ldots, a_{n}$ independently by the function $\operatorname{random}(0,1)$, i.e., $a_{u}=$ $\operatorname{random}(0,1) \forall u \in\left\{m_{k+1}, m_{k+2}, \ldots, n\right\}$

Step 4.5 Assign $a_{1}, a_{2}, \ldots, a_{m_{k}}$ at the location $[i, j]$ of the random grids corresponding to the participants of selected set $B_{m_{k}}^{t}$, i.e.,

$$
\begin{aligned}
& R_{p_{1}^{t}}[i, j]=a_{1} \\
& R_{p_{2}^{t}}[i, j]=a_{2} \\
& \quad \vdots \\
& \quad \vdots \\
& R_{p_{m_{k}}^{t}}[i, j]=a_{m_{k}}
\end{aligned}
$$

Step 4.6 Assign $a_{m_{k+1}}, a_{m_{k+2}}, \ldots, a_{n}$ randomly at the location $[i, j]$ of the remaining $\left(n-m_{k}\right)$ random grids

Step 4.7 Repeat Step 4.1 until all the pixels $A[i, j]$ of the secret image $A$ are encrypted

Step 4.8 output $\left(R_{1}, R_{2}, \ldots, R_{n}\right)$

\subsection{Scheme for color images}

The proposed scheme can be easily extended to color images by adopting a similar procedure as discussed in [4] 5]. A color model, which is either additive (RGB) or subtractive (CMY), is employed to decompose the color image into three channels. The procedure to encrypt a color secret image $B$ for general access structures is given as follows:

Step 5.1 Decompose the color secret image $B$ into three color components Cyan, Magenta and Yellow (CMY), i.e., $B^{C}, B^{M}$ and $B^{Y}$

Step 5.2 By using error diffusion halftone techniques [10, 15], transform the color components $B^{C}, B^{M}$ and $B^{Y}$ into halftone images, i.e., $H B^{C}, H B^{M}$ and $H B^{Y}$

Step 5.3 Generate $n$ cipher grids for each of halftone color components $H B^{C}, H B^{M}$ and $H B^{Y}$ by using the proposed scheme for binary images, i.e., $R_{i}^{C}, R_{i}^{M}$ and $R_{i}^{Y}$ where $1 \leq i \leq n$

Step 5.4 The color components of the cipher grids $R_{i}^{C}, R_{i}^{M}$ and $R_{i}^{Y}$ are combined to form eight color cipher grid $R_{i}$, i.e., $R_{i}=$ $\left(R_{i}^{C}, R_{i}^{M}, R_{i}^{Y}\right)$ where $1 \leq i \leq n$

Step 5.5 output $\left(R_{1}, R_{2}, \ldots, R_{n}\right)$

\section{PERFORMANCE ANALYSIS}

The performance of the proposed scheme is measured in terms of the security of the original image and the visual quality of the reconstructed image.

DEFINITION 1. For a certain pixel $r$ in a binary image $R$ of the size $h \times w$, the light transmission of $r(t(r))$ is defined as the probability of $r$ to be transparent (i.e., $\operatorname{Prob}(r=0)$ ). Thus, the light transmission of a transparent (respectively opaque) pixel $r \in R$ is $t(r)=1$ (respectively $t(r)=0$ ). Additionally, the average light transmission of $R$ is defined as $T(R)=$ $\frac{1}{h \times w} \sum_{i=1}^{h} \sum_{j=1}^{w} t(R[i, j])$.

In random-grid based VSS, the visual quality of the reconstructed image is measured by the contrast [13], which is defined as follows.

DEFINITION 2. The contrast of the image $S$ reconstructed for the binary image $A$ is defined as $\alpha=\frac{T(S[A(0)])-T(S[A(1)])}{1+T(S[A(1)])}$. $A(0)$ (respectively $A(1)$ ) denotes the area of all transparent (respectively opaque) pixels in $A$, with $A=A(0) \cup A(1)$ and $A(0) \cap A(1)=\emptyset . S[A(0)]$ (respectively $S[A(1)]$ ) denotes the area of all pixels in $S$ corresponding to $A(0)$ (respectively $A(1)$ ).

DEFINITION 3. For the contrast $\alpha>0$, the reconstructed image $S$ visually reveals the original image $A$. Precisely, $\alpha>0$ implies $T(S[A(0)])>T(S[A(1)])$ and $S$ is visually recognizable as A. For $\alpha=0($ i.e. $T(S[A(0)])=T(S[A(1)])), S$ is meaningless and reveals no information about $A$.

If $R$ is a random grid, then for $r \in R$, the probability of $r$ to be transparent (0) is equal to the probability of $r$ to be opaque (1), i.e., $\operatorname{Prob}(r=0)=\operatorname{Prob}(r=1)=\frac{1}{2}$. Since the number of transparent pixels is probabilistically equal to that of opaque pixels in $R$, we have $T(R)=\frac{1}{2}$. Let $\otimes$ denote Boolean OR operation, which simulates the human visual system.

LEMMA 4. If $r_{1}, r_{2}, \ldots, r_{n}$ are $n$ random pixels generated independently by the function random $(0,1)$, then $\operatorname{Prob}\left(r_{1} \otimes r_{2} \otimes\right.$ $\left.\cdots \otimes r_{n}=0\right)=\frac{1}{2^{n}}$.

PROOF. We prove by mathematical induction on $n$. We have $\operatorname{Prob}\left(r_{1} \otimes r_{2}=0\right)=\operatorname{Prob}\left(r_{1}=0\right) \times \operatorname{Prob}\left(r_{2}=0\right)=\frac{1}{2} \times \frac{1}{2}=$ $\frac{1}{2^{2}}$, i.e., the result is true for $n=2$.

Assume that the result holds for $n-1$, i.e., $\operatorname{Prob}\left(r_{1} \otimes \ldots \otimes r_{n-1}=\right.$ $0)=\frac{1}{2^{n-1}}$. We have to prove that it holds for $n$ also. We know that $\operatorname{Prob}\left(r_{1} \otimes \ldots \otimes r_{n-1} \otimes r_{n}=0\right)=\operatorname{Prob}\left(r_{1} \otimes \ldots \otimes r_{n-1}=\right.$ $0) \times \operatorname{Prob}\left(r_{n}=0\right)=\frac{1}{2^{n-1}} \times \frac{1}{2}=\frac{1}{2^{n}}$. 
LEMMA 5. If $r_{1}, r_{2}, \ldots, r_{n}$ are $n$ random pixels generated independently by the function random $(0,1)$, then $\operatorname{Prob}\left(r_{1} \oplus r_{2} \oplus\right.$ $\left.\ldots \oplus r_{n}=0\right)=\frac{1}{2}$.

Proof. We prove by mathematical induction on $n$. We have $\operatorname{Prob}\left(r_{1} \oplus r_{2}=0\right)=\operatorname{Prob}\left(r_{1}=0\right) \times \operatorname{Prob}\left(r_{2}=0\right)+$ $\operatorname{Prob}\left(r_{1}=1\right) \times \operatorname{Prob}\left(r_{2}=1\right)=\left(\frac{1}{2} \times \frac{1}{2}\right)+\left(\frac{1}{2} \times \frac{1}{2}\right)=\frac{1}{2}$, i.e., the result is true for $n=2$.

Assume that the result holds for $n-1$, i.e., $\operatorname{Prob}\left(r_{1} \oplus \ldots \oplus r_{n-1}=\right.$ $0)=\frac{1}{2}$. We have to prove that it holds for $n$ also. We know that $\operatorname{Prob}\left(r_{1} \oplus \ldots \oplus r_{n-1} \oplus r_{n}=0\right)=\operatorname{Prob}\left(r_{1} \oplus \ldots \oplus r_{n-1}=\right.$ $0) \times \operatorname{Prob}\left(r_{n}=0\right)+\operatorname{Prob}\left(r_{1} \oplus \ldots \oplus r_{n-1}=1\right) \times \operatorname{Prob}\left(r_{n}=\right.$ 1) $=\left(\frac{1}{2} \times \frac{1}{2}\right)+\left(\frac{1}{2} \times \frac{1}{2}\right)=\frac{1}{2}$.

THEOREM 6. In the proposed random-grid based VSS scheme for general access structures, each cipher grid is meaningless and reveals no information about the secret image $A$.

Proof. In the proposed scheme, each pixel $A[i, j] \in A$ is encrypted corresponding to a minimal qualified set randomly selected from the basis $\Gamma_{0}$. Let $A[i, j]$ be encrypted corresponding to a minimal qualified set of cardinality $m_{k}$, where $k \in\{1,2, \ldots, r\}$. Therefore, the pixel values at location $[i, j]$ of the cipher grids $R_{1}, R_{2}, \ldots, R_{n}$ (i.e., $R_{1}[i, j], R_{2}[i, j], \ldots, R_{n}[i, j]$ ) are selected among the binary values $a_{1}, a_{2}, \ldots, a_{m_{k}-1}, a_{m_{k}}, a_{m_{k}+1}, \ldots, a_{n}$ that are generated by Steps 4.3 to 4.4 of the proposed scheme. Since the binary values $a_{1}, a_{2}, \ldots, a_{m_{k}-1}, a_{m_{k}+1}, \ldots, a_{n}$ are generated independently by the function $\operatorname{random}(0,1), \operatorname{Prob}\left(a_{l}=0\right)=\frac{1}{2}$ for $1 \leq l \leq m_{k-1}$ and $m_{k+1} \leq l \leq n$

From Step 4.3.2 of the proposed scheme, we have $b_{m_{k}-1}=a_{1} \oplus$ $a_{2} \oplus \ldots \oplus a_{m_{k}-1}$. By Lemma 5, we obtain

$$
\operatorname{Prob}\left(b_{m_{k}-1}=0\right)=\frac{1}{2} .
$$

As $a_{m_{k}}$ depends on the algorithm selected from Algorithms 1-3, three cases are considered as follows.

In Algorithm 1, for $A[i, j]=0, a_{m_{k}}=b_{m_{k}-1}$, i.e, $\operatorname{Prob}\left(a_{m_{k}}=\right.$ $0)=\operatorname{Prob}\left(b_{m_{k}-1}=0\right)=\frac{1}{2}$. While for $A[i, j]=1, a_{m_{k}}=$ $\overline{b_{m_{k}-1}}$, i.e., $\operatorname{Prob}\left(a_{m_{k}}=0\right)=1-\operatorname{Prob}\left(b_{m_{k}-1}=0\right)=\frac{1}{2}$. Therefore, $\operatorname{Prob}\left(a_{m_{k}}=0\right)=\frac{1}{2}$.

In Algorithm 2, for $A[i, j]=0, a_{m_{k}}=b_{m_{k}-1}$, i.e., $\operatorname{Prob}\left(a_{m_{k}}=\right.$ $0)=\operatorname{Prob}\left(b_{m_{k}-1}=0\right)=\frac{1}{2}$. While for $A[i, j]=1$, $a_{m_{k}}=\operatorname{random}(0,1)$, i.e., $\operatorname{Prob}\left(a_{m_{k}}=0\right)=\frac{1}{2}$. Therefore, $\operatorname{Prob}\left(a_{m_{k}}=0\right)=\frac{1}{2}$

In Algorithm 3, for $A[i, j]=0, a_{m_{k}}=\operatorname{random}(0,1)$, i.e., $\operatorname{Prob}\left(a_{m_{k}}=0\right)=\frac{1}{2}$. While for $A[i, j]=1, a_{m_{k}}=\overline{b_{m_{k}-1}}$, i.e., $\operatorname{Prob}\left(a_{m_{k}}=0\right)=1-\operatorname{Prob}\left(b_{m_{k}-1}=0\right)=\frac{1}{2}$. Therefore, $\operatorname{Prob}\left(a_{m_{k}}=0\right)=\frac{1}{2}$.

Precisely, no matter if $A[i, j]=0$ or 1 , we obtain $\operatorname{Prob}\left(R_{l}[i, j]=\right.$ $0)=\frac{1}{2}$, i.e., $t\left(R_{l}[i, j]\right)=\frac{1}{2}$ for $1 \leq l \leq n$. Therefore,

$$
T\left(R_{l}[A(0)]\right)=T\left(R_{l}[A(1)]\right)=\frac{1}{2},
$$

where $1 \leq l \leq n$. Hence, each cipher grid is meaningless and does not reveal any information about the secret image $A$.

THEOREM 7. In the proposed random-grid based VSS scheme for general access structures, stacking the cipher grids corresponding to the participants of any forbidden set cannot reveal the secret image $A$.

Proof. Let $F=\left\{p_{1}, p_{2}, \ldots, p_{s}\right\}$ be any forbidden set and $S$ denote the stacking result of the cipher grids corresponding to the participants of $F$, i.e., $S[i, j]=R_{p_{1}}[i, j] \otimes R_{p_{2}}[i, j] \otimes \ldots \otimes$ $R_{p_{s}}[i, j]$, where $1 \leq i \leq h$ and $1 \leq j \leq w$. We define

$$
C_{F}=\left\{Q \in \Gamma_{0}: F \subset Q\right\} .
$$

In the proposed scheme, each pixel $A[i, j] \in A$ is encrypted corresponding to a minimal qualified set, which is selected from the basis $\Gamma_{0}$ randomly. Let $A[i, j]$ be encrypted corresponding to a minimal qualified set $Q_{1} \in \Gamma_{0}$. Two cases are considered, where Case 1 is for $Q_{1} \notin C_{F}$ or $C_{F}=\emptyset$, and Case 2 is for $Q_{1} \in C_{F}$.

Case 1: In this case, for $A[i, j]=0$ or 1 , the pixels $R_{p_{1}}[i, j], R_{p_{2}}[i, j], \ldots, R_{p_{s}}[i, j]$ are generated independently as random values. By Lemma 4 , no matter if $A[i, j]=0$ or 1 , we obtain $\operatorname{Prob}(S[i, j]=0)=\frac{1}{2^{s}}$, i.e., $t(S[i, j])=\frac{1}{2^{s}}$. Therefore,

$$
T(S[A(0)])=T(S[A(1)])=\frac{1}{2^{s}} .
$$

Case 2: Let $\left|Q_{1}\right|=m_{k}$ for some $k \in\{1,2, \ldots, r\}$. Since $F \subset Q_{1}$ and $Q_{1} \in \Gamma_{0}$, the pixel values at any location $[i, j]$ of the cipher grids corresponding to the participants of $F$ will be assigned from the set $\left\{a_{1}, a_{2}, \ldots, a_{m_{k}}\right\}$ generated as in Step 4.3 of the proposed scheme. If $D=\left\{R_{p_{1}}[i, j], R_{p_{2}}[i, j], \ldots, R_{p_{s}}[i, j]\right\}$, then $D \subset$ $\left\{a_{1}, a_{2}, \ldots, a_{m_{k}}\right\}$. Considering $a_{m_{k}}$ as the last value generated based on selection from Algorithms 1-3, we have either $a_{m_{k}} \notin D$ or $a_{m_{k}} \in D$.

If $a_{m_{k}} \notin D$, then $D \subseteq\left\{a_{1}, a_{2}, \ldots, a_{m_{k}-1}\right\}$. We know that $a_{1}, a_{2}, \ldots, a_{m_{k}-1}$ are generated independently by the function random $(0,1)$. By using Lemma 4 , for $A[i, j]=0$ or 1 , we obtain $\operatorname{Prob}(S[i, j]=0)=\frac{1}{2^{s}}$, i.e., $t(S[i, j])=\frac{1}{2^{s}}$. Therefore,

$$
T(S[A(0)])=T(S[A(1)])=\frac{1}{2^{s}} .
$$

If $a_{m_{k}} \in D$, then consider $R_{p_{u}}[i, j]=a_{m_{k}}$ for some $u \in\{1,2, \ldots, s\}$. If $D_{1}=D \backslash\left\{R_{p_{u}}[i, j]\right\}=$ $\left\{R_{y_{1}}[i, j], \ldots, R_{y_{s-1}}[i, j]\right\}$, then each pixel $R_{y_{v}}[i, j] \in D_{1}$ is generated independently by the function $\operatorname{random}(0,1)$, i.e. $\operatorname{Prob}\left(R_{y_{v}}[i, j]=0\right)=\frac{1}{2}$ for $1 \leq v \leq s-1$. By Lemma 4 , we obtain

$$
\operatorname{Prob}\left(R_{y_{1}}[i, j] \otimes \ldots \otimes R_{y_{s-1}}[i, j]=0\right)=\frac{1}{2^{s-1}} .
$$

We know that $R_{y_{1}}[i, j] \otimes \ldots \otimes R_{y_{s-1}}[i, j]$ will be transparent (0) only if

$$
R_{y_{1}}[i, j]=\ldots \ldots=R_{y_{s-1}}[i, j]=0 .
$$

If $R_{y_{v}}[i, j]=0(1 \leq v \leq s-1)$, then $b_{m_{k}-1}=$ $R_{z_{1}}[i, j] \oplus \ldots \oplus R_{z_{m_{k}-s}}[i, \bar{j}]$, where $\left\{R_{z_{1}}[i, j], \ldots, R_{z_{m_{k}-s}}[i, j]\right\}$ $=\left\{a_{1}, a_{2}, \ldots, a_{m_{k}-1}\right\} \backslash D_{1}$. By using Lemma 5 , we obtain

$$
\operatorname{Prob}\left(b_{m_{k}-1}=0\right)=\frac{1}{2} \text {. }
$$

We have $\operatorname{Prob}(S[i, j]=0)=\operatorname{Prob}\left(R_{y_{1}}[i, j] \otimes \ldots \otimes\right.$ $\left.R_{y_{s-1}}[i, j]=0\right) \times \operatorname{Prob}\left(R_{p_{u}}[i, j]=0\right)$. Therefore,

$$
\operatorname{Prob}(S[i, j]=0)=\frac{1}{2^{s-1}} \times \operatorname{Prob}\left(R_{p_{u}}[i, j]=0\right) .
$$

The pixel $R_{p_{u}}[i, j]$ (= $a_{m_{k}}$ ) depends upon $b_{m_{k}-1}$ and the algorithm selected from Algorithms 1-3.

In Algorithm 1, for $A[i, j]=0$, we have $R_{p_{u}}[i, j]=b_{m_{k}-1}$, i.e., $\operatorname{Prob}\left(R_{p_{u}}[i, j]=0\right)=\operatorname{Prob}\left(b_{m_{k}-1}=0\right)=\frac{1}{2}$. From 3 , we obtain $\operatorname{Prob}(S[i, j]=0)=\frac{1}{2^{s-1}} \times \frac{1}{2}=\frac{1}{2^{s}}$, i.e., $t(S[i, j])=\frac{1}{2^{s}}$. Therefore,

$$
T(S[A(0)])=\frac{1}{2^{s}}
$$

In addition, for $A[i, j]=1$, we have $R_{p_{u}}[i, j]=\overline{b_{m_{k}-1}}$, i.e., $\operatorname{Prob}\left(R_{p_{u}}[i, j]=0\right)=1-\operatorname{Prob}\left(b_{m_{k}-1}=0\right)=\frac{1}{2}$. From 3 , we obtain $\operatorname{Prob}(S[i, j]=0)=\frac{1}{2^{s-1}} \times \frac{1}{2}=\frac{1}{2^{s}}$, i.e., $t(S[i, j])=\frac{1}{2^{s}}$. Therefore,

$$
T(S[A(1)])=\frac{1}{2^{s}} .
$$


From (4) and (5), we have

$$
T(S[A(0)])=T(S[A(1)])=\frac{1}{2^{s}} .
$$

In Algorithm 2, for $A[i, j]=0$, we have $R_{p_{u}}[i, j]=b_{m_{k}-1}$. We obtain

$$
T(S[A(0)])=\frac{1}{2^{s}} .
$$

In addition, for $A[i, j]=1$, we have $R_{p_{u}}[i, j]=\operatorname{random}(0,1)$, i.e., $\operatorname{Prob}\left(R_{p_{u}}[i, j]=0\right)=\frac{1}{2}$. From 3, we obtain $\operatorname{Prob}(S[i, j]=0)=\frac{1}{2^{s-1}} \times \frac{1}{2}=\frac{1}{2^{s}}$, i.e., $t(S[i, j])=\frac{1}{2^{s}}$. Therefore,

$$
T(S[A(1)])=\frac{1}{2^{s}} .
$$

From (7) and (8), we have

$$
T(S[A(0)])=T(S[A(1)])=\frac{1}{2^{s}} .
$$

In Algorithm 3, for $A[i, j]=0$, we have $R_{p_{a}}[i, j]=$ $\operatorname{random}(0,1)$, i.e., $\operatorname{Prob}\left(R_{p_{u}}[i, j]=0\right)=\frac{1}{2}$. From (3), we obtain $\operatorname{Prob}(S[i, j]=0)=\frac{1}{2^{s-1}} \times \frac{1}{2}=\frac{1}{2^{s}}$, i.e., $t(S[i, j])=\frac{1}{2^{s}}$. Therefore,

$$
T(S[A(0)])=\frac{1}{2^{s}} .
$$

In addition, for $A[i, j]=1$, we have $R_{p_{u}}[i, j]=\overline{b_{m_{k}-1}}$. We obtain

$$
T(S[A(1)])=\frac{1}{2^{s}} .
$$

From $(10)$ and 11 , we have

$$
T(S[A(0)])=T(S[A(1)])=\frac{1}{2^{s}} .
$$

Precisely in Case 2, from (6), 9) and (12), we have

$$
T(S[A(0)])=T(S[A(1)])=\frac{1}{2^{s}} .
$$

By considering both cases (1) and (13), we obtain

$$
T(S[A(0)])=T(S[A(1)]) .
$$

By Definition 3, we obtain $\alpha=0$. Thus, $S$ is meaningless and reveals no information about $A$.

THEOREM 8. In the proposed random-grid based VSS scheme for general access structures, the secret image A can be visually revealed by stacking the cipher grids corresponding to the participants of any qualified set.

Proof. Let $Q=\left\{p_{1}, p_{2}, \ldots, p_{t}\right\}$ be any qualified set consisting of $t$ participants and $S$ denote the stacking result of the cipher grids corresponding to the participants of $Q$. We have $S[i, j]=$ $R_{p_{1}}[i, j] \otimes R_{p_{2}}[i, j] \otimes \ldots \otimes R_{p_{t}}[i, j]$, where $1 \leq i \leq h$ and $1 \leq j \leq w$. Let $C_{Q}$ be the set of minimal qualified sets which are the subsets of $Q$ and belong to the basis $\Gamma_{0}$, i.e.,

$$
C_{Q}=\left\{Q_{1} \subseteq Q: Q_{1} \in \Gamma_{0}\right\},
$$

and $c=\left|C_{Q}\right|$.

In the proposed scheme, each pixel $A[i, j] \in A$ is encrypted corresponding to a minimal qualified set, which is selected from the basis $\Gamma_{0}$ randomly. Let $A[i, j]$ be encrypted corresponding to a minimal qualified set $Q_{1} \in \Gamma_{0}$. Two cases are considered, where Case 1 is for $Q_{1} \notin C_{Q}$ and Case 2 is for $Q_{1} \in C_{Q}$. In Case 2, we have

$$
\operatorname{Prob}\left(Q_{1} \in C_{Q}\right)=\frac{c}{N},
$$

and in Case 1, we have

$$
\operatorname{Prob}\left(Q_{1} \notin C_{Q}\right)=1-\frac{c}{N},
$$

where $N=\left|\Gamma_{0}\right|$. By considering Case 1 and Case 2, we have

$$
\begin{aligned}
\operatorname{Prob}(S[i, j]= & 0)=\left(1-\frac{c}{N}\right) \times \operatorname{Prob}(S[i, j]=0 \mid \text { Case } 1)+ \\
& \left(\frac{c}{N}\right) \times \operatorname{Prob}(S[i, j]=0 \mid \text { Case } 2) .
\end{aligned}
$$

Case 1: In this case, the pixels $R_{p_{1}}[i, j], R_{p_{2}}[i, j], \ldots, R_{p_{t}}[i, j]$ are generated randomly so that they are independent of corresponding secret pixel $A[i, j]$, i.e., $\operatorname{Prob}\left(R_{p_{l}}[i, j]=0\right)=\frac{1}{2}$ for $1 \leq l \leq t$. By using Lemma 4 , for $A[i, j]=0$ or 1 , we obtain

$$
\operatorname{Prob}(S[i, j]=0 \mid \text { Case } 1)=\frac{1}{2^{t}} .
$$

Case 2: Let $D=\left\{R_{p_{1}}[i, j], R_{p_{2}}[i, j], \ldots, R_{p_{t}}[i, j]\right\}$ and $\left|Q_{1}\right|=m_{k}$ for some $k \in\{1,2, \ldots, r\}$. The pixels $R_{p_{1}}[i, j], R_{p_{2}}[i, j], \ldots, R_{p_{t}}[i, j]$ will be selected from the set $\left\{a_{1}, a_{2}, \ldots, a_{m_{k}}, a_{m_{k+1}}, \ldots, a_{n}\right\}$ generated as in Steps 4.3 to 4.4 of the proposed scheme. Let us assume that

$$
D_{1}=\left\{R_{y_{1}}[i, j], \ldots, R_{y_{m_{k}}}[i, j]\right\}=\left\{a_{1}, \ldots, a_{m_{k}}\right\},
$$

and

$$
D_{2}=\left\{R_{z_{1}}[i, j], \ldots, R_{z_{t-m_{k}}}[i, j]\right\} \subseteq\left\{a_{m_{k}+1}, \ldots, a_{n}\right\},
$$

where $D_{1} \subseteq D, D_{2} \subseteq D, D_{1} \cap D_{2}=\emptyset$ and $D=D_{1} \cup D_{2}$. We have $\operatorname{Prob}(S[i, j]=\overline{0} \mid$ Case 2$)=\operatorname{Prob}\left(R_{p_{1}}[i, j] \otimes R_{p_{2}}[i, j] \otimes\right.$ $\left.\ldots \otimes R_{p_{t}}[i, j]=0\right)=\operatorname{Prob}\left(R_{y_{1}}[i, j] \otimes \ldots \otimes R_{y_{m_{k}}}[i, j]=\right.$ $0) \times \operatorname{Prob}\left(R_{z_{1}}[i, j] \otimes \ldots \otimes R_{z_{t-m_{k}}}[i, j]=0\right)$.

The binary values $a_{m_{k}+1}, a_{m_{k}+2}, \ldots, a_{n}$ are generated independently by the function random $(0,1)$. By Lemma 4 , we obtain

$$
\operatorname{Prob}\left(R_{z_{1}}[i, j] \otimes \ldots \otimes R_{z_{t-m_{k}}}[i, j]=0\right)=\frac{1}{2^{t-m_{k}}} .
$$

In addition, we have $\operatorname{Prob}\left(R_{y_{1}}[i, j] \otimes \ldots \otimes R_{y_{m_{k}}}[i, j]=0\right)=$ $\operatorname{Prob}\left(a_{1} \otimes a_{2} \otimes \ldots \otimes a_{m_{k}-1} \otimes a_{m_{k}}=0\right)=\operatorname{Prob}\left(a_{1} \otimes a_{2} \otimes\right.$ $\left.\ldots \otimes a_{m_{k}-1}=0\right) \times \operatorname{Prob}\left(a_{m_{k}}=0\right)$.

The binary values $a_{1}, a_{2}, \ldots, a_{m_{k}-1}$ are generated independently by the function random $(0,1)$. By Lemma 4 ,

$$
\operatorname{Prob}\left(a_{1} \otimes a_{2} \otimes \ldots \otimes a_{m_{k}-1}=0\right)=\frac{1}{2^{m_{k}-1}} .
$$

We know that $a_{1} \otimes a_{2} \otimes \ldots \otimes a_{m_{k}-1}$ will be transparent (0) only if $a_{1}, a_{2}, \ldots, a_{m_{k}-1}$ are transparent, i.e., $a_{1}=a_{2}=\ldots=$ $a_{m_{k}-1}=0$. By Step 4.3.2 of the proposed scheme, for $a_{v}=0$ $\left(1 \leq v \leq m_{k}-1\right)$, we obtain $b_{m_{k}-1}=0$. The value of $a_{m_{k}}$ depends on $b_{m_{k}-1}$ and the algorithm selected from Algorithms 1-3. In Algorithm 1, for $A[i, j]=0$, we have $a_{m_{k}}=b_{m_{k}-1}=0$, i.e., $\operatorname{Prob}\left(a_{m_{k}}=0\right)=1$. Therefore,

$\operatorname{Prob}\left(R_{y_{1}}[i, j] \otimes \ldots \otimes R_{y_{m_{k}}}[i, j]=0\right)=\frac{1}{2^{m_{k}-1}} \times 1=\frac{1}{2^{m} k^{-1}}$.

We obtain

$$
\operatorname{Prob}(S[i, j]=0 \mid \text { Case } 2)=\frac{1}{2^{m_{k}-1}} \times \frac{1}{2^{t-m_{k}}}=\frac{1}{2^{t-1}} .
$$

For $A[i, j]=0$, by considering both cases $[14)$ and $[15$, , we have $\operatorname{Prob}(S[i, j]=0)=\left(1-\frac{c}{N}\right) \times \frac{1}{2^{t}}+\left(\frac{c}{N}\right) \times \frac{1}{2^{t-1}}=\left(1+\frac{c}{N}\right) \times \frac{1}{2^{t}}$, i.e., $t(S[i, j])=\left(1+\frac{c}{N}\right) \times \frac{1}{2^{t}}$. Therefore,

$$
T(S[A(0)])=\left(1+\frac{c}{N}\right) \times \frac{1}{2^{t}} .
$$

In addition, for $A[i, j]=1$ we have $a_{m_{k}}=\overline{b_{m_{k}-1}}=1$, i.e., $\operatorname{Prob}\left(a_{m_{k}}=0\right)=0$. Therefore,

$$
\operatorname{Prob}\left(R_{y_{1}}[i, j] \otimes \ldots \otimes R_{y_{m_{k}}}[i, j]=0\right)=\frac{1}{2^{m_{k}-1}} \times 0=0 .
$$


We obtain

$$
\operatorname{Prob}(S[i, j]=0 \mid \text { Case } 2)=0 \times \frac{1}{2^{t-m_{k}}}=0 .
$$

For $A[i, j]=1$, by considering both cases 14 and $[16$, we have $\operatorname{Prob}(S[i, j]=0)=\left(1-\frac{c}{N}\right) \times \frac{1}{2^{t}}+\left(\frac{c}{N}\right) \times 0=\left(1-\frac{c}{N}\right) \times \frac{1}{2^{t}}$, i.e., $t(S[i, j])=\left(1-\frac{c}{N}\right) \times \frac{1}{2^{t}}$. Therefore,

$$
T(S[A(1)])=\left(1-\frac{c}{N}\right) \times \frac{1}{2^{t}} .
$$

Thus, the contrast of $S$ is

$$
\begin{aligned}
\alpha & =\frac{T(S[A(0)])-T(S[A(1)])}{1+T(S[A(1)])}=\frac{\left(1+\frac{c}{N}\right) \times \frac{1}{2^{t}}-\left(1-\frac{c}{N}\right) \times \frac{1}{2^{t}}}{1+\left(1-\frac{c}{N}\right) \times \frac{1}{2^{t}}} \\
& =\frac{2 \times c}{N \times 2^{t}+N-c} .
\end{aligned}
$$

In Algorithm 2, for $A[i, j]=0$, we have $a_{m_{k}}=b_{m_{k}-1}=0$. By the following similarly as in case of Algorithm 1, we obtain

$$
T(S[A(0)])=\left(1+\frac{c}{N}\right) \times \frac{1}{2^{t}} .
$$

For $A[i, j]=1$, we have $a_{m_{k}}=\operatorname{random}(0,1)$, i.e., $\operatorname{Prob}\left(a_{m_{k}}=\right.$ $0)=\frac{1}{2}$. Therefore,

$\operatorname{Prob}\left(R_{y_{1}}[i, j] \otimes \ldots \otimes R_{y_{m_{k}}}[i, j]=0\right)=\frac{1}{2^{m_{k}-1}} \times \frac{1}{2}=\frac{1}{2^{m_{k}}}$.

We obtain

$$
\operatorname{Prob}(S[i, j]=0 \mid \text { Case } 2)=\frac{1}{2^{m_{k}}} \times \frac{1}{2^{t-m_{k}}}=\frac{1}{2^{t}} .
$$

For $A[i, j]=1$, by considering both cases (14) and (18), we have

$$
\operatorname{Prob}(S[i, j]=0)=\left(1-\frac{c}{N}\right) \times \frac{1}{2^{t}}+\left(\frac{c}{N}\right) \times \frac{1}{2^{t}}=\frac{1}{2^{t}},
$$

i.e., $t(S[i, j])=\frac{1}{2^{t}}$. Therefore,

$$
T(S[A(1)])=\frac{1}{2^{t}} .
$$

Thus, the contrast of $S$ is

$$
\alpha=\frac{T(S[A(0)])-T(S[A(1)])}{1+T(S[A(1)])}=\frac{\left(1+\frac{c}{N}\right) \times \frac{1}{2^{t}}-\frac{1}{2^{t}}}{1+\frac{1}{2^{t}}}=\frac{c}{N \times 2^{t}+N} .
$$

In Algorithm 3, for $A[i, j]=0$, we have $a_{m_{k}}=\operatorname{random}(0,1)$, i.e., $\operatorname{Prob}\left(a_{m_{k}}=0\right)=\frac{1}{2}$. Therefore,

$\operatorname{Prob}\left(R_{y_{1}}[i, j] \otimes \ldots \otimes R_{y_{m_{k}}}[i, j]=0\right)=\frac{1}{2^{m_{k}-1}} \times \frac{1}{2}=\frac{1}{2^{m_{k}}}$.

We obtain

$$
\operatorname{Prob}(S[i, j]=0 \mid \text { Case } 2)=\frac{1}{2^{m_{k}}} \times \frac{1}{2^{t-m_{k}}}=\frac{1}{2^{t}} .
$$

For $A[i, j]=0$, by considering both cases $(14)$ and 20 , we obtain

$$
\operatorname{Prob}(S[i, j]=0)=\left(1-\frac{c}{N}\right) \times \frac{1}{2^{t}}+\left(\frac{c}{N}\right) \times \frac{1}{2^{t}}=\frac{1}{2^{t}},
$$

i.e., $t(S[i, j])=\frac{1}{2^{t}}$. Therefore,

$$
T(S[A(0)])=\frac{1}{2^{t}} .
$$

For $A[i, j]=1$, we have $a_{m_{k}}=\overline{b_{m_{k}-1}}=1$. By the following similarly as in case of Algorithm 1, we obtain

$$
T(S[A(1)])=\left(1-\frac{c}{N}\right) \times \frac{1}{2^{t}} .
$$

Thus, the contrast of $S$ is

$$
\alpha=\frac{T(S[A(0)])-T(S[A(1)])}{1+T(S[A(1)])}=\frac{\frac{1}{2^{t}}-\left(1-\frac{c}{N}\right) \times \frac{1}{2^{t}}}{1+\left(1-\frac{c}{N}\right) \times \frac{1}{2^{t}}}=\frac{c}{N \times 2^{t}+N-c} .
$$

From (17), 19 and $\sqrt{21}$, we have $\alpha>0$ (i.e. $T(S[A(0)])>$ $T(S[A(1)]))$ in the proposed scheme based on Algorithms 1-3. Thus by Definition 3, the stacked image $S$ visually reveals the original secret image $A$.

\section{EXPERIMENTAL RESULTS AND COMPARISON WITH RELATED WORK}

For experiments and comparison, Algorithm 1 is selected from Algorithms 1-3 as required in Step 4.3.2 of the proposed scheme. The proposed scheme is experimented for binary and color images by considering two access structures defined on a set $\mathcal{P}=\{1,2,3,4\}$ of four participants.

Access structure 1: Basis $\Gamma_{0}=\{\{1,2\},\{1,3\},\{2,3\}\}, \Gamma_{\text {Qual }}=$ $\{\{1,2\},\{1,3\},\{2,3\},\{1,2,3\},\{1,2,4\},\{1,3,4\},\{2,3,4\},\{1,2$, $3,4\}\}$ and $\Gamma_{\text {Forb }}=\{\{1\},\{2\},\{3\},\{4\},\{1,4\},\{2,4\},\{3,4\}\}$. Access structure 2: Basis $\Gamma_{0}=\{\{1,4\},\{2,3,4\}\}, \Gamma_{\text {Qual }}=$ $\{\{1,4\},\{1,2,4\},\{1,3,4\},\{2,3,4\},\{1,2,3,4\}\}$ and $\Gamma_{\text {Forb }}=$ $\{\{1\},\{2\},\{3\},\{4\},\{1,2\},\{1,3\},\{2,3\},\{2,4\},\{3,4\},\{1,2,3\}\}$.

\subsection{Experiment 1}

This experiment is conducted for the secret image of size $1024 \times$ 1024 shown in Fig. 1(a). The secret image is encrypted into four cipher grids (Figs. 1(b)-1(e)) for the access structure 1. Each cipher grid is meaningless and does not reveal the secret information. By the stacked images for the forbidden sets (Figs. 1(h), 1(j), 1(k)), no secret information can be revealed. The stacked images for the qualified sets (Figs. 1(f), 1(g), 1(i), 1(l)-1(p)) can visually reveal the secret image. Table 1 shows the contrast of the stacked images for the qualified sets of the access structure 1 .

\begin{tabular}{|c|c|c|c|c|}
\hline Scheme & $(2,2)$ & $(n, n)$ & $(k, n)$ & Access structure \\
\hline Kafri and Keren [6] & $\frac{1}{2}$ & - & - & - \\
\hline Shyu [13] & $\frac{1}{2}$ & $\frac{1}{2^{n-1}}$ & - & - \\
\hline Chen and Tsao [4] & $\frac{1}{2}$ & $\frac{1}{2^{n-1}}$ & 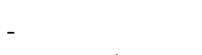 & - \\
\hline Chen and Tsao [5] & $\frac{1}{2}$ & $\frac{1}{2^{n-1}}$ & $\frac{2 \times\left(\begin{array}{l}t \\
k\end{array}\right)}{\left(2^{t}+1\right) \times\left(\begin{array}{l}n \\
k\end{array}\right)-\left(\begin{array}{l}t \\
k\end{array}\right)}$ & - \\
\hline Ours & $\frac{1}{2}$ & $\frac{1}{2^{n-1}}$ & $\frac{2 \times\left(\begin{array}{l}t \\
k\end{array}\right)}{\left(2^{t}+1\right) \times\left(\begin{array}{l}n \\
k\end{array}\right)-\left(\begin{array}{l}t \\
k\end{array}\right)}$ & $\frac{2 \times c}{N \times 2^{t}+N-c}$ \\
\hline
\end{tabular}

\subsection{Experiment 2}

In this experiment, a color image of size $1024 \times 1024$ shown in Fig. 2(a) is encrypted into four cipher grids (Figs. 2(b)-2(e)) for the access structure 2. Each cipher grid is meaningless and reveals no secret information. The stacked images for the forbidden sets (Figs. 2(f), 2(g), 2(i)-2(l)) do not reveal any information about the secret image. The secret image can be easily recognized by the stacked images for the qualified sets (Figs. 2(h), 2(m)-2(p))

Table 1. Contrast of the stacked images for the qualified sets of the access structure 1

\begin{tabular}{llll}
\hline Qualified set & Contrast $\alpha$ & Qualified set & Contrast $\alpha$ \\
\hline$\{1,2\}$ & $1 / 7$ & $\{1,2,4\}$ & $1 / 13$ \\
$\{1,3\}$ & $1 / 7$ & $\{1,3,4\}$ & $1 / 13$ \\
$\{2,3\}$ & $1 / 7$ & $\{2,3,4\}$ & $1 / 13$ \\
$\{1,2,3\}$ & $1 / 4$ & $\{1,2,3,4\}$ & $1 / 8$ \\
\hline
\end{tabular}

Table 2. Comparison of the contrast between the proposed scheme and random-grid based VSS schemes

$k \leq t \leq n$. 


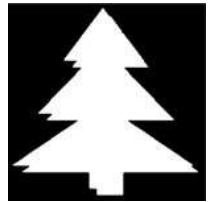

(a)

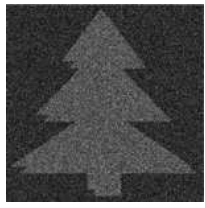

(i)

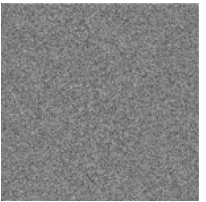

(b)

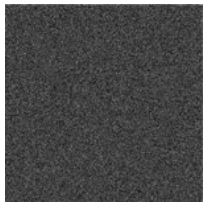

(j)

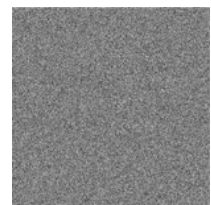

(c)

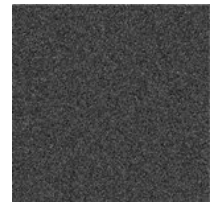

(k)

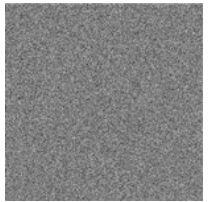

(d)

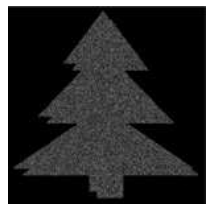

(1)

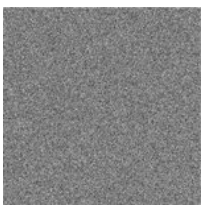

(e)

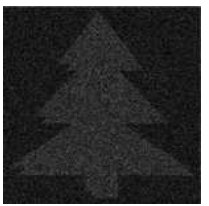

(m)

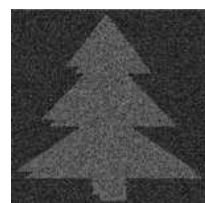

(f)

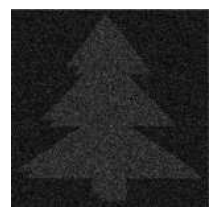

(n)

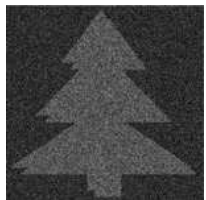

(g)

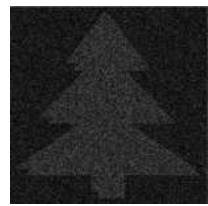

(o)

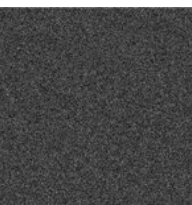

(h)

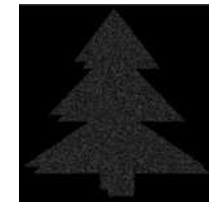

(p)

Fig. 1 The experimental results of the proposed scheme for the access structure 1: (a) Binary secret image; (b) $R_{1}$; (c) $R_{2}$; (d) $R_{3}$; (e) $\mathbf{R}_{4}$; (f) $\mathbf{R}_{1} \otimes \mathbf{R}_{2}$; (g) $\mathbf{R}_{1} \otimes \mathbf{R}_{3}$; (h) $\mathbf{R}_{1} \otimes \mathbf{R}_{4}$; (i) $\mathbf{R}_{2} \otimes \mathbf{R}_{3}$; (j) $\mathbf{R}_{2} \otimes \mathbf{R}_{4}$; (k) $\mathbf{R}_{3} \otimes \mathbf{R}_{4}$; (l) $\mathbf{R}_{1} \otimes \mathbf{R}_{2} \otimes \mathbf{R}_{3}$; (m) $\mathbf{R}_{1} \otimes \mathbf{R}_{2} \otimes \mathbf{R}_{4}$; (n) $\mathbf{R}_{1} \otimes \mathbf{R}_{3} \otimes \mathbf{R}_{4} ;(\mathbf{o}) \mathbf{R}_{2} \otimes \mathbf{R}_{3} \otimes \mathbf{R}_{4} ;(\mathbf{p}) \mathbf{R}_{1} \otimes \mathbf{R}_{2} \otimes \mathbf{R}_{3} \otimes \mathbf{R}_{4}$.

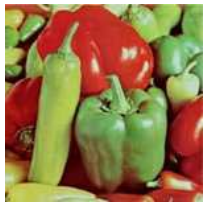

(a)

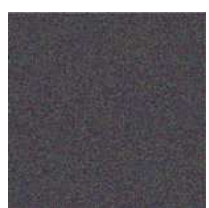

(i)

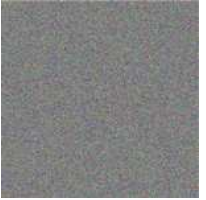

(b)

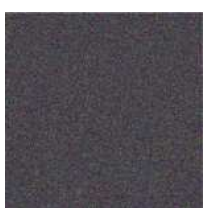

(j)

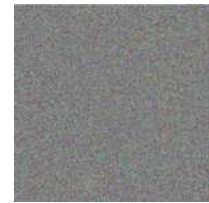

(c)

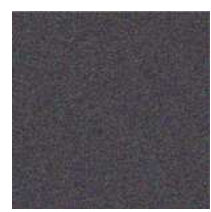

(k)

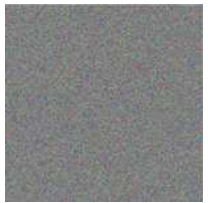

(d)

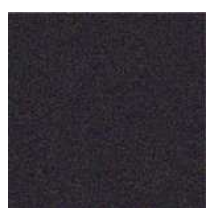

(1)

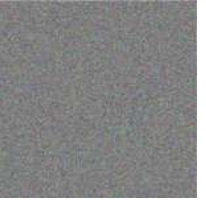

(e)

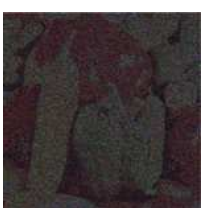

$(\mathrm{m})$

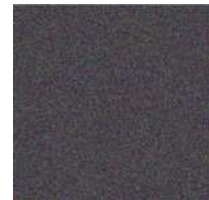

(f)

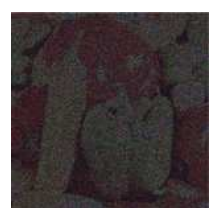

(n)

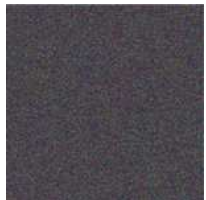

(g)

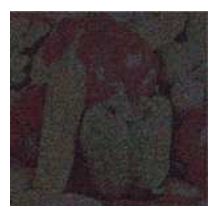

(o)

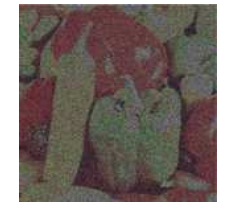

(h)

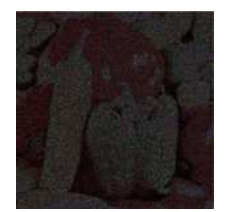

(p)

Fig. 2 The experimental results of the proposed scheme for the access structure 2: (a) Color secret image; (b) $R_{1}$; (c) $R_{2}$; (d) $R_{3}$; (e) $\mathbf{R}_{4}$; (f) $\mathbf{R}_{1} \otimes \mathbf{R}_{2}$; (g) $\mathbf{R}_{1} \otimes \mathbf{R}_{3}$; (h) $\mathbf{R}_{1} \otimes \mathbf{R}_{4}$; (i) $\mathbf{R}_{2} \otimes \mathbf{R}_{3}$; (j) $\mathbf{R}_{2} \otimes \mathbf{R}_{4}$; (k) $\mathbf{R}_{3} \otimes \mathbf{R}_{4}$; (l) $\mathbf{R}_{1} \otimes \mathbf{R}_{2} \otimes \mathbf{R}_{3}$; (m) $\mathbf{R}_{1} \otimes \mathbf{R}_{2} \otimes \mathbf{R}_{4}$; (n) $\mathbf{R}_{1} \otimes \mathbf{R}_{3} \otimes \mathbf{R}_{4} ;(\mathbf{o}) \mathbf{R}_{2} \otimes \mathbf{R}_{3} \otimes \mathbf{R}_{4} ;(\mathbf{p}) \mathbf{R}_{1} \otimes \mathbf{R}_{2} \otimes \mathbf{R}_{3} \otimes \mathbf{R}_{4}$.

Table 3. Comparison of the contrast between the proposed scheme and Wu and Sun's scheme [16]

\begin{tabular}{lll}
\hline Qualified set & The proposed scheme & Wu and Sun's scheme [16] \\
\hline$\{1,2\}$ & $1 / 7$ & $1 / 19$ \\
$\{2,4\}$ & $1 / 7$ & $1 / 19$ \\
$\{1,2,3\}$ & $1 / 13$ & $1 / 17$ \\
$\{1,2,4\}$ & $4 / 25$ & $1 / 19$ \\
$\{2,3,4\}$ & $1 / 13$ & $1 / 17$ \\
$\{1,3,4\}$ & $1 / 13$ & $1 / 17$ \\
$\{1,2,3,4\}$ & $1 / 8$ & $1 / 17$ \\
\hline
\end{tabular}

\subsection{Comparison with related work}

A random-grid based $(k, n)$-threshold VSS scheme with $2 \leq k \leq$ $n$ can be obtained as a special case of the proposed scheme by taking the basis $\Gamma_{0}=\{Q \subseteq \mathcal{P}:|Q|=k\}$. In this case, we have $c=\left(\begin{array}{l}t \\ k\end{array}\right)$ and $N=\left(\begin{array}{l}n \\ k\end{array}\right)$ for any qualified set of $t(\geq k)$ participants. Thus, the contrast (in Algorithm 4 based on Algorithm 1) of the image obtained by stacking any $t$ shares is $\frac{2 \times\left(\begin{array}{l}t \\ k\end{array}\right)}{\left(2^{t}+1\right) \times\left(\begin{array}{l}n \\ k\end{array}\right)-\left(\begin{array}{l}t \\ k\end{array}\right)}$.
Table 4. Comparison of the contrast between the proposed scheme and Shyu's scheme [14]

\begin{tabular}{llll}
\hline Qualified set & $\begin{array}{l}\text { The proposed } \\
\text { scheme }\end{array}$ & $\begin{array}{l}\text { Shyu's method } \\
\text { by using } \Gamma_{0}[14]\end{array}$ & $\begin{array}{l}\text { Shyu's method } \\
\text { by using } Z_{M}[14]\end{array}$ \\
\hline$\{1,2,3\}$ & $2 / 35$ & $1 / 256$ & $1 / 32$ \\
$\{1,2,4\}$ & $2 / 35$ & $1 / 256$ & $1 / 32$ \\
$\{1,3,4\}$ & $2 / 35$ & $1 / 256$ & $1 / 32$ \\
$\{2,3,4\}$ & $2 / 35$ & $1 / 256$ & $1 / 32$ \\
$\{1,2,3,4\}$ & $1 / 8$ & $1 / 256$ & $1 / 32$ \\
\hline
\end{tabular}

This is same as the contrast obtained in random-grid based $(k, n)$ threshold VSS scheme [5]. If we take $t=n$, then the contrast of the decoded image will be $\frac{1}{2^{n-1}}$, which is again same as obtained in random-grid based $(n, n)$ VSS scheme [4, 13]. The proposed scheme reconstructs the secret image with visual quality similar to random-grid based VSS schemes [4, 6, 13] as shown in Table 2 Similar to our scheme, the VSS schemes [14, 16] can also handle general access structures. To compare the proposed scheme with $\mathrm{Wu}$ and Sun's scheme [16], an access structure defined on a set 
Table 5. Comparison of the proposed scheme with related VSS schemes

\begin{tabular}{|c|c|c|c|c|c|}
\hline Scheme & $\begin{array}{l}\text { Encoding } \\
\text { based on }\end{array}$ & Type & $\begin{array}{l}\text { Pixel } \\
\text { expansion }\end{array}$ & $\begin{array}{l}\text { Codebook } \\
\text { requirement }\end{array}$ & $\begin{array}{l}\text { Secret image } \\
\text { format }\end{array}$ \\
\hline Naor and Shamir [11] & Basis matrices & $(n, n)$ and $(k, n)$ & Yes & Yes & Binary \\
\hline Ateniese et al. [2] & Basis matrices & Access structure & Yes & Yes & Binary \\
\hline Adhikari et al. 1] & Basis matrices & Access structure & Yes & Yes & Binary \\
\hline Wu and Sun 16 & Basis matrices & Access structure & No & Yes & Binary and Color \\
\hline Kafri and Keren 6 & Random-grid & $(2,2)$ & No & No & Binary \\
\hline Shyu 13 & Random-grid & $(n, n)$ & No & No & Binary and Color \\
\hline Chen and Tsao 4 & Random-grid & $(2, n)$ and $(n, n)$ & No & No & Binary and Color \\
\hline Chen and Tsao [5] & Random-grid & $(k, n)$ & No & No & Binary and Color \\
\hline Ours & Random-grid & Access structure & No & No & Binary and Color \\
\hline
\end{tabular}

$\mathcal{P}=\{1,2,3,4\}$ with the basis $\Gamma_{0}=\{\{1,2\},\{2,4\},\{1,3,4\}\}$ and $Z_{M}=\{\{1,3\},\{1,4\},\{2,3\},\{3,4\}\}$ is taken. The following modified basis matrices are obtained by using the method discussed in [16], which are used to generate the shares.

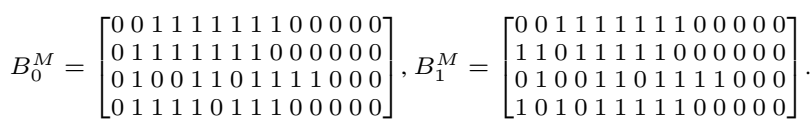

Table 3 shows the contrast of the decoded images in the proposed scheme and $\mathrm{Wu}$ and Sun's scheme [16] for the given access structure. It is obvious that the proposed scheme can achieve higher contrast compared to Wu and Sun's scheme [16]. Shyu's scheme [14] adopts two different algorithms for encrypting a secret image into general access structures, i.e., by using the construction based on either basis $\Gamma_{0}$ or collection of maximal forbidden sets $Z_{M}$. For comparison between the proposed scheme and Shyu's scheme [14], the access structure defined on a set $\mathcal{P}=\{1,2,3,4\}$ with the basis $\Gamma_{0}=\{\{1,2,3\},\{1,2,4\},\{1,3,4\}\{2,3,4\}\}$ and $Z_{M}=$ $\{\{1,2\},\{1,3\},\{1,4\},\{2,3\},\{2,4\},\{3,4\}\}$ is considered. Table 4 confirms that the proposed scheme can achieve higher contrast while comparing to algorithms proposed in [14].

In Table 5 the proposed scheme is compared with the related VSS schemes in terms of the pixel expansion, codebook requirement, type of the scheme and the secret image format. Compared to VSS schemes [1, 2, 11], the proposed scheme benefits by sharing binary as well as color images without any pixel expansion and codebook requirement. Compared to the proposed scheme, Wu and Sun's scheme [16] can share the secret image for general access structures without any pixel expansion, but requires the basis matrices before encryption process. The proposed scheme generalizes the existing random-grid based VSS schemes [4, 6, 13] to share a secret image for general access structures. Precisely, the proposed scheme for general access structures is obtained by extending the random-grid based algorithm and attains the security conditions perfectly, i.e., only qualified sets can recover the secret image while the forbidden sets cannot gain any information about the secret image.

\section{CONCLUSION}

In this paper, a VSS scheme for general access structures is designed based on random grids. The proposed scheme can be used to share a secret image into general access structures without any pixel expansion and codebook requirement. The security analysis and experimental results are given to confirm that the proposed scheme performs well. The potential applications of the proposed scheme may include image sharing, visual authentication, digital watermarking, image hiding, etc.

\section{References}

[1] A. Adhikari, T. K. Dutta, and B. Roy. A new black and white visual cryptographic scheme for general access structures. In Indocrypt'04, volume 3348, pages 399-413. LNCS, SpringerVerlag, 2004.

[2] G. Ateniese, C. Blundo, A. De Santis, and D. R. Stinson. Visual cryptography for general access structures. Information and Computation, 129:86-106, 1996.

[3] G. R. Blakley. Safeguarding cryptographic keys. AFIPS Conference Proceedings, 48:313-317, 1979.

[4] T. H. Chen and K. H. Tsao. Visual secret sharing by random grids revisited. Pattern Recognition, 42:2203-2217, 2009.

[5] T. H. Chen and K. H. Tsao. Threshold visual secret sharing by random grids. The Journal of Systems and Software, 84:11971208, 2011.

[6] O. Kafri and E. Keren. Encryption of pictures and shapes by random grids. Optics Letters, 12(6):377-379, 1987.

[7] S. Kumar and R. K. Sharma. Improving contrast in random grids based visual secret sharing. International Journal of Security and Its Application, 6:9-28, 2012.

[8] S. Kumar and R. K. Sharma. Recursive information hiding of secrets by random grids. Cryptologia, 37:154-161, 2013.

[9] S. Kumar and R. K. Sharma. Threshold visual secret sharing based on boolean operations. Security and Communication Networks, 2013. dOI:10.1002/sec.769.

[10] D. L. Lau and G. R. Arce. Modern Digital Halftoning. Marcel Dekker, New York, 2000.

[11] M. Naor and A. Shamir. Visual cryptography. In Proceedings of Advances in Cryptology (EUROCRYPT 94), volume 950, pages 1-12. LNCS,Springer-Verlag, 1995.

[12] A. Shamir. How to share a secret. Communication of the ACM, 22(11):612-613, 1979.

[13] S. J. Shyu. Image encryption by multiple random grids. Pattern Recognition, 42:1582-1596, 2009.

[14] S. J. Shyu. Visual cryptograms of random grids for general access structures. IEEE Trans. on Circuits and Systems for Video Technology, 23:414-424, 2013.

[15] R. Ulichney. Digital Halftoning. The MIT Press, Cambridge, 1987.

[16] X. Wu and W. Sun. Random grid-based visual secret sharing for general access structures with cheat-preventing ability. The Journal of Systems and Software, 85:1119-1134, 2012. 